Editor's Note: These short, critical reviews of recent papers in the Journal, written exclusively by graduate students or postdoctoral fellows, are intended to summarize the important findings of the paper and provide additional insight and commentary. For more information on the format and purpose of the Journal Club, please see http://www.jneurosci.org/misc/ifa_features.shtml.

\title{
Weighting Pain Avoidance and Reward Seeking: A Neuroeconomical Approach to Pain
}

\author{
Mathieu Roy \\ Department of Psychology, Columbia University, New York, New York 10027 \\ Review of Talmi et al.
}

The most important choices that we have to make in life often entail both positive and negative consequences. As common wisdom suggests and as formalized in many economic theories, the best choice of action is the one that maximizes rewards and pleasure and minimizes losses and pain. However, the way that positive and negative outcomes are weighted against one another within our brains is only beginning to be unraveled by the emerging discipline of neuroeconomics. We believe that the findings yielded by this scientific enterprise will not only prove to be useful for the understanding of our decision-making processes, but will also shed light on the way the value of pain is represented in our brains and how it influences our behavior.

In a recent neuroimaging experiment, Talmi et al. (2009) examined the effects of pain on reward sensitivity in a task in which rewards were paired with painful electrical stimulations. For each trial, participants had to choose between two options bearing either a high (75\%) or low (25\%) probability of receiving an electrical stimulation. On each trial, one of two possible pairs of options was presented to the participants. For one pair of options, the intensity of the stimulation outcome was always set at a non-

Received Jan. 16, 2010; revised Feb. 5, 2010; accepted Feb. 16, 2010. I thank the "Fonds de Recherche en Santé du Québec" for a postdoctoral scholarship and Joshua Haag for English revision.

Correspondence should be addressed to Mathieu Roy, Department of Psychology, Columbia University, 1190 Amsterdam Avenue, New York, NY 10027.E-mail:mr2973@columbia.edu.

DOI:10.1523/JNEUROSCI.0262-10.2010

Copyright $\odot 2010$ the authors $\quad 0270-6474 / 10 / 304185-02 \$ 15.00 / 0$ painful level, whereas for the other, electrical stimulations were always set at a painful level. The probabilities of the stimulation outcomes associated with each option were learned beforehand in a pre-experimental session. During the experimental session, electrical stimulations became systematically paired with monetary rewards. The value of the reward at stake for each trial was displayed at the beginning of the trial along with the pair of options. For the trials with nonpainful outcomes, participants simply chose the option with the highest probability of reward. However, for the trials with painful outcomes, participants increasingly chose the low-probability option as the value of monetary reward decreased, indicating an interaction between pain and reward [Talmi et al. (2009), their Fig. 2]. Brain imaging results showed that this effect was paralleled by a reduced activation in rewardrelated regions of the brain, such as the ventral striatum and the rostral anterior cingulate cortex (rACC), when participants were deciding which option to choose [Talmi et al. (2009), their Fig. 4]. Thus, the prospect of pain rendered rewards less desirable and decreased reward-related activity in the brain.

Interestingly, activation of right anterior insula (raINS) during pain trials was larger among participants for whom the interactive effect of pain on choice behavior was stronger [Talmi et al. (2009), their Fig. 5]. This finding of a correlation between raINS activity and pain avoidance behavior is consistent with the results of previous studies implicating the anterior insula in the anticipation and prediction of pain. Moreover, results from other studies suggest that the avoidance function of the raINS is not limited to pain, but also is associated with other forms of aversive events, such as monetary losses. For instance, right insula activation was shown to predict, on a trial-by-trial basis, the deterrent effects of high prices on purchasing decisions (Knutson et al., 2007).

These findings also fit nicely with recent models of interoception and emotional awareness. According to one of these models (Craig, 2009), the foundation for our emotional feelings lies in the neural representation of the physiological condition of the body in the insular cortex. Raw interoceptive signals coding for the homeostatic status of the body, such as pain, would first project to the posterior insula and become progressively integrated with contextual motivational and hedonic information as they progress toward the anterior insula. The culminating point of this progression would be a complex and rich representation of the "global emotional moment" in the anterior insula. In accordance with the "somatic marker" hypothesis of Damasio (1993), this meta-representation of bodily states would constitute an emotional feeling, accessible to consciousness and providing the "gut-feeling" that often guides our decision processes. In the case of the experiment of Talmi et al. (2009), the raINS activation might have represented the bad feeling associated with pain anticipation that discouraged participants from choosing the high-probability option when rewards were low. In support of this interpretation, skin conductance responses in pain trials were higher for 
participants whose choices were more influenced by the interactive effects of pain, linking choice behavior to physiological arousal.

The involvement of the anterior insula, and specifically the raINS, in the anticipation of aversive events in general led some researchers to propose a key role for this structure in affective disorders. Moreover, increased raINS activation during pain anticipation was found to predict higher pain responses in depressed patients vs controls (Strigo et al., 2008). It is therefore not surprising to see that patients with idiopathic chronic pain disorders, who often exhibit anxiodepressive symptoms, also show increased pain-related activation in the raINS (Gündel et al., 2008). Indeed, a key finding in the study of pain-related disabilities is that pain-related fear is a far better predictor of pain-related disability than pain intensity itself. Thus, the interindividual differences in the effects of pain on reward sensitivity observed by Talmi et al. (2009) could very well reflect interindividual differences in pain-related fear, especially since the subjective intensity of the painful stimulation was set constant across participants. As a matter of fact, one of the most debilitating consequences of chronic pain is the withdrawal from previously valued activities to avoid pain. Consequently, one of the targets of psychotherapy for chronic pain is to help patient weight the value of their painavoiding behavior against that of the abandoned pleasurable activities.

To persuade people to accept aversive consequences, it is common practice to offer them a compensatory benefit. In fact, this is exactly what happened to the participants in the experiment of Talmi et al. (2009) when they switched from a pre-experimental conditioning session, in which the painful stimulus was not accompanied by any reward, to the experimental session where pain and reward were paired. The inclusion of rewards in the experimental session had a dramatic effect on their choice behavior: the participants, who had chosen the low-probability option $82 \%$ of the time during the preexperimental conditioning session, switched to choose the high-probability option almost $80 \%$ of the time to get the newly introduced reward.

This almost instantaneous shift in choice behavior after the inclusion of rewards is highly suggestive of a goal-directed process. Goal-directed processes, which are sensitive to feedback about goal values, are often distinguished from actions that have become relatively automatic or habitual after an extended period of learning. It is quite probable that the pain-avoidance choices in the experiment of Talmi et al. (2009) were ren- dered somewhat habitual as a result of extensive learning during the preexperimental conditioning session. The persistence of these automatic pain-avoiding choices during the experimental session could explain in part the interactive effect of pain on the appetitive, goal-directed choices instantiated by the explicit inclusion of rewards. A more systematic exploration of this hypothesis could be relevant to our understanding of chronic pain and pain-related disabilities, as these have often been attributed to an inflexible learning of the contingencies associated with pain. In doing so, pain researchers would adopt a strategy that has proven quite fruitful in the study of many psychiatric disorders, such as obsessive-compulsive disorder and drug addiction.

The identification of the brain systems involved in overcoming pain avoidance by rewarding stimuli would also improve our understanding of endogenous pain-regulatory mechanisms. Unfortunately, the experimental design of Talmi et al. (2009) was not well suited to answer that question. Nevertheless, the finding that the nucleus accumbens (NA) and the rACC integrated the values of pain and gain makes these structures well positioned to regulate pain avoidance tendencies in the face of pursuable rewards. Indeed, ACC-lesioned animals are disinclined to exert effort to obtain higher rewards, even though they have no deficit in the valuation of these rewards per se. (Walton et al., 2003). Strikingly, the rACC is also repeatedly shown to be involved in the antinociceptive effects of cognitive interventions, such as distraction or placebo analgesia. This is particularly interesting since recent accounts of placebo analgesia underline the key role of motivational factors and brain structures associated with motivation, such as the NA and the subgenual and rACC, in the generation of placebo effects (Scott et al., 2008). Altogether, these findings point to the involvement of the NA and rACC in the overcoming of pain when it interferes with the pursuit of more valued, meaningful goals.

Interestingly, personality traits associated with dopaminergic neurotransmission, such as novelty seeking, behavioral drive, fun seeking, reward responsiveness, and harm avoidance (which is inversely related to dopamine), were recently shown to predict the amplitude of placebo analgesia, as well as NA gray-matter density (Schweinhardt et al., 2009). Moreover, genes involved in dopaminergic neurotransmission have also been shown to influence pain sensitivity. For instance, a polymorphism of the catechol-O-methyl-transferase (COMT) gene coding for high activity of the dopa- mine-regulating COMT enzyme has been linked to reduced pain sensitivity (Zubieta et al., 2003). Conversely, polymorphisms coding for low activity of the COMT enzyme, along with pain catastrophizing, have recently been shown to predict the occurrence of postoperative pain (George et al., 2008). A better understanding of the manner in which these genetic and psychological predispositions impact the valuation of pain and rewards as well as their interaction in the brain could help explain why individuals react so differently to pain. Experimental paradigms developed within the framework of neuroeconomics, such as the one developed by Talmi et al. (2009), may prove highly useful in this endeavor and might bring meaningful insights to our current understanding of chronic pain and painrelated disabilities.

\section{References}

Craig AD (2009) How do you feel-now? The anterior insula and human awareness. Nat Rev Neurosci 10:59-70.

Damasio AR (1993) Descartes' error: emotion, reason, and the human brain. New York: Putnam.

George SZ, Wallace MR, Wright TW, Moser MW, Greenfield WH 3rd, Sack BK, Herbstman DM, Fillingim RB (2008) Evidence for a biopsychosocial influence on shoulder pain: pain catastrophizing and catechol-O-methyltransferase (COMT) diplotype predict clinical pain ratings. Pain 136:53-61.

Gündel H, Valet M, Sorg C, Huber D, Zimmer C, Sprenger T, Tölle TR (2008) Altered cerebral response to noxious heat stimulation in patients with somatoform pain disorder. Pain 137:413-421.

Knutson B, Rick S, Wimmer GE, Prelec D, Loewenstein G (2007) Neural predictors of purchases. Neuron 53:147-156.

Schweinhardt P, Seminowicz DA, Jaeger E, Duncan GH, Bushnell MC (2009) The anatomy of the mesolimbic reward system: a link between personality and the placebo analgesic response. J Neurosci 29:4882-4887.

Scott DJ, Stohler CS, Egnatuk CM, Wang H, Koeppe RA, Zubieta JK (2008) Placebo and nocebo effects are defined by opposite opioid and dopaminergic responses. Arch Gen Psychiatry 65: 220-231.

Strigo IA, Simmons AN, Matthews SC, Craig AD, Paulus MP (2008) Association of major depressive disorder with altered functional brain response during anticipation and processing of heat pain. Arch Gen Psychiatry 65:1275-1284.

Talmi D, Dayan P, Kiebel SJ, Frith CD, Dolan RJ (2009) How humans integrate the prospects of pain and reward during choice. J Neurosci 29:14617-14626.

Walton ME, Bannerman DM, Alterescu K, Rushworth MF (2003) Functional specialization within medial frontal cortex of the anterior cingulate for evaluating effort-related decisions. J Neurosci 23:6475-6479.

Zubieta JK, Heitzeg MM, Smith YR, Bueller JA, Xu K, Xu Y, Koeppe RA, Stohler CS, Goldman D (2003) COMT val158met genotype affects muopioid neurotransmitter responses to a pain stressor. Science 299:1240-1243. 